\title{
Intratumor Heterogeneity of EGFR Activating Mutations in Advanced NSCLC Patients at Single-Cell Level
}

\author{
Liu Yuefen \\ Dongying City People's Hospital Shandong, Dongying 257091
}

Keywords: EGFR, NSCLC, Intratumor Heterogeneity

\begin{abstract}
Introduction: Present study is to explore the controversial problem whether the intratumoral heterogeneity of EGFR activating mutation exists at the level of single cancer cells. Materials and Methods: Single H1975 cells harbored EGFR exon 21 L858R mutation were isolated by flow cytometry to assess the feasibility of single-cell analysis for EGFR exon 21 by nested polymerase chain reaction and derect sequence. Then, six patients diagnosed with lung adenocarcinoma whose fresh frozen specimens harbored EGFR exon 21 mutation tested by direct sequencing were chosen. All of them received gefitnib treatment and the PFS of three patients was longer than 12 months (Group A) while the PFS of other three patients was shorter than 6 months (Group B). By using the established method based on single H1975 cells, EGFR exon 21 mutational status was analyzed in single tumor cells which were captured from tumor sample by Laser Capture Microdissection. Results: A total of 104 individual H1975 cells were obtained. The amplification rate and allele drop-out rate were $96.2 \%$ and $7.0 \%$, respectively. A total of 135 tumor cells from six patients' samples were captured. The amplification rate of nested PCR was 84.3\% (59/70) in Group A and 93.8\% (61/65) in Group B ( $P=0.077)$. The mutational rate was $86.4 \%$ in Group A, which was significantly higher than the total mutational rate $68.9 \%$ in Group $\mathrm{B}(P=0.021)$.Conclusion: The intratumoral heterogeneity of EGFR activating mutation in lung adenocarcinoma does exist based on the analysis of single cancer cells.
\end{abstract}

\section{Introduction}

Lung cancer has become a leading cause of cancer-related death all over the world ${ }^{1}$. In recent years, several large randomized controlled clinical studies consistently demonstrated that epidermal growth factor receptor (EGFR) tyrosine kinase inhibitors (TKIs) have shown great efficacy in the treatment of non-small cell lung cancer (NSCLC) patients with EGFR activating mutation compared with chemotherapy in first line treatments ${ }^{2-6}$. However, the response to EGFR-TKIs is quite different in EGFR mutant patients. Some patients experienced longer progression-free survival (PFS) of more than 1 year, whereas some had PFS of shorter than 6 months. Our previous study showed that the relative EGFR mutation abundance in tumor tissues could predict benefit from EGFR-TKI treatments ${ }^{7}$. [However, the study demonstrated EGFR heterogeneity based on the tissue level] To our knowledge, phenotypical and functional heterogeneity among cancer cells that might be associated with the response to therapy has long been proposed and accepted ${ }^{8}$. Recently, driver gene EGFR activating mutational heterogeneity, including intratumoral heterogeneity, intertumoral heterogeneity and pre- or post-treatment heterogeneity ${ }^{9}$ was considered as a potential cause of diverse response to EGFR-TKIs ${ }^{10-12}$. However, heterogeneity of EGFR activating mutation and its effect of EGFR-TKIs still remains divergent viewpoint in NSCLC partients ${ }^{13-15}$.

The methods in above studies used for detecting EGFR status have been established on complex mixture of cells or tissue level. Therefore, misinterpretation for EGFR mutational information might occur due to interference of cell populations. Single-cell analysis was direct and better understanding of genetic characteristics of tumors by flow cytometry (FCM) and Laser Capture Microdissection (LCM $)^{16}$. Single tumor cell analysis might provide a deeper insight into the occurrence of intratumoral EGFR activating mutation heterogeneity ${ }^{17}$.

In the present study, single H1975 cells which harbors EGFR L858R heterozygous mutation in exon 21 were isolated by FCM were to evaluate the feasibility of single-cell analysis for EGFR 
mutation detection. Based on the method, we investigated the occurrence of intratumoral EGFR activating mutational heterogeneity at the level of single tumor cells in lung adenocarcenoma which had EGFR 21 exon L858R mutation.

\section{Materials and Methods}

\subsection{Cell culture}

NSCLC cell line H1975 has L858R heterozygous mutation in exon 21 of the EGFR gene ${ }^{18}$. It was kindly presented by professor Tony S. Mok (Prince of Wales Hospital, Hong Kong) in our study. He purchased H1975 cell line from American Type Culture Collection (ATCC). H1975 cells were cultured on flasks coated with RPMI 1640 containing $10 \%$ fetal calf serum and incubated at $37^{\circ} \mathrm{C}$ in a humidified atmosphere with $5 \% \mathrm{CO}_{2}$. When the cells grew well to $80 \% \sim 90 \%$ of the flasks, we digested them with the use of trypsinase to prepare single cell suspension for isolation.

\subsection{Tissue samples}

In this study, six patients with advanced lung adenocarcinoma harbored EGFR exon 21 L858R mutation under gefitnib treatment in their late stage were screened (Table 1). They have the following clinical characteristics: They were all treated in Guangdong General Hospital and enough tissue preserved at the tumor tissue biobank of the Guangdong Lung Cancer Institute(GLCI); Pathology of their tumors showed pure adenocarcinoma and EGFR L858R mutation in exon 21 by direct sequencing; In their first-line treatment they were all treated with gefitnib. Their progression-free survival (PFS) of EGFR-TKIs was 22, 19, 15, 5, 3, 1 months , respectively. The 3 of patient which PFS longer than 12 months was screened as long-term PFS group, and selected 3 patients which PFS of shorter than 6 months as short-term PFS group. All the patients had written informed consent.

Table 1. The clinicopathologic feature of six patients

\begin{tabular}{cccccccccccc}
\hline $\begin{array}{c}\text { Patient } \\
\text { no. }\end{array}$ & Sex & Age & PS & $\begin{array}{c}\text { Smoking } \\
\text { history }\end{array}$ & Stage & Tumor & Histology & $\begin{array}{c}\text { EGFR } \\
\text { status }\end{array}$ & $\begin{array}{c}\text { TKI } \\
\text { treatment }\end{array}$ & $\begin{array}{c}\text { PFS } \\
\text { Response to } \\
\text { TKIs }\end{array}$ \\
\hline 3647 & male & 87 & 1 & 1 & IV & Lung & Ad & L858R & First-line & 19 & PR \\
2715 & male & 47 & 2 & 0 & IV & Lung & Ad & L858R & First-line & 22 & SD \\
4128 & male & 42 & 2 & 1 & IV & Lung & Ad & L858R & First-line & 15 & PR \\
3669 & male & 76 & 1 & 1 & IV & Lung & Ad & L858R & First-line & 5 & SD \\
1813 & male & 60 & 2 & 0 & IV & Lung & Ad & L858R & First-line & 3 & PD \\
3651 & female & 64 & 2 & 0 & IV & Lung & Ad & L858R & First-line & 1 & PD \\
\hline
\end{tabular}

* PS: ECOG performance status; Ad: adenocarcinoma

\subsection{Single cell isolation and DNA extraction}

H1975 cell suspension was prepared to isolate single H1975 cell into 96-well plates added with 10ul cell lysis solution in each well $(50 \mathrm{mmol} / \mathrm{L}$ Tris, $1 \mathrm{mmol} / \mathrm{L}$ EDTA, 0.5\% Tween-20, 200mg/L proteinase K) by using flow cytometry (FCM, Becton Dickinson, BD).

For each frozen tissue sample, a 5 - $\mu$ m-thick section was mounted on a membrane slide (Arcturus ${ }^{\mathrm{XT}}$ ) and then stained with hematoxylin for histomorphologic identification of cells. Dehydration steps were performed: $70 \%$ ethanol for 30s, $95 \%$ ethanol for 30s, and $100 \%$ ethanol for 30s. Then the slides were put in xylene through a $5 \mathrm{~min}$ bath to remove Ethanol. After air-dried, the sections were microdissected to capture individual tumor cells incorporating infrared(IR) laser capture systems and ultraviolet(UV) laser cutting systems in LCM(Arcturus ${ }^{\mathrm{XT}}$ ) instrument according to the standard LCM protocol. In each tumor section 20 - 24 tumor cells were captured. Figure 1 showed the sites where the single tumor cells captured in tumor section roughly.[]In Arcturus $^{\mathrm{XT}}$ system, ultraviolet laser cut the wanted single tumor cells, whereas infrared laser melt 
thermolabile polymers for cell capture. Following microdissection, we added the cell lysis solution directly to the polymer film and insert the polymer end of the cap into the top of a $500 \mu \mathrm{L}$ microcentrifuge tube allowing the collection of the whole cell lysates for downstream analysis (Arcturus). ${ }^{19}$

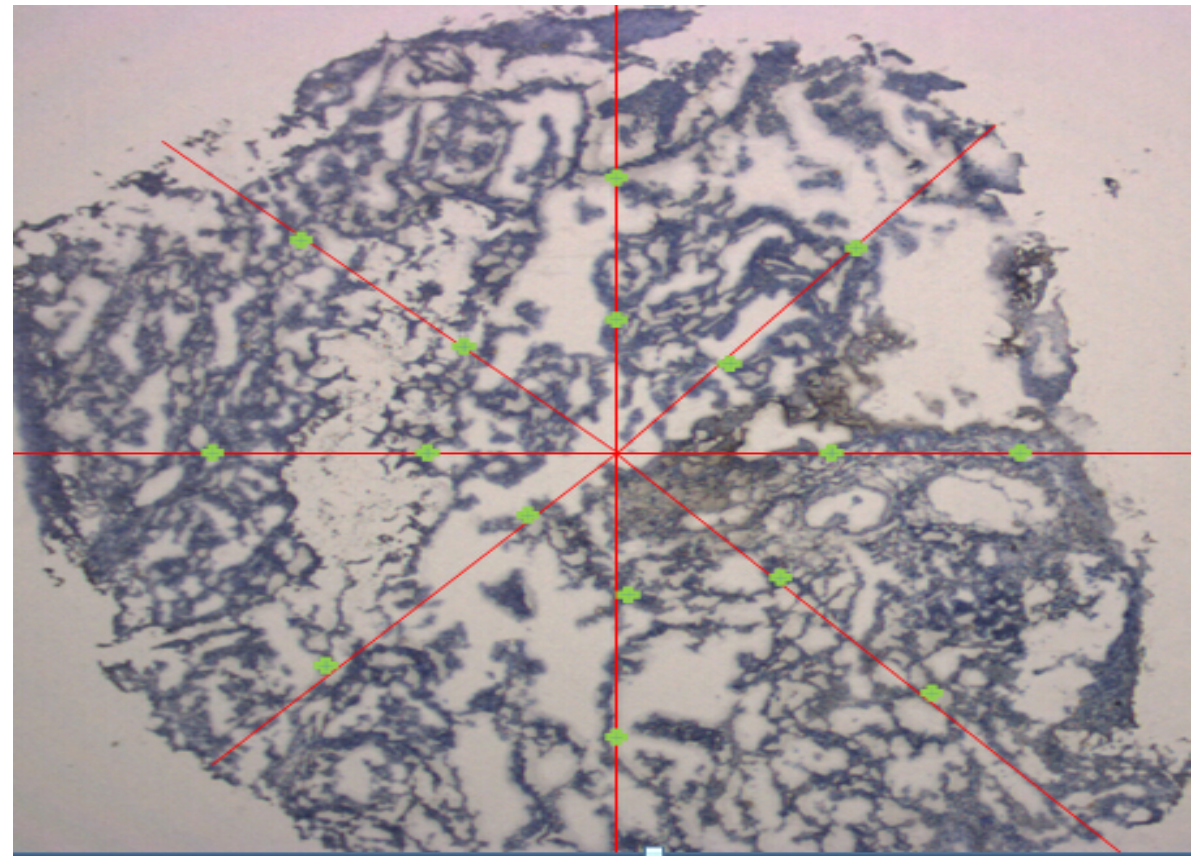

Figure 1. Single tumor cell captured map in one section

(Green crosses represented the sites of single tumor cells captured along the red lines roughly.)

\subsection{Single cell PCR analysis}

The whole DNA extracted from single cell was submitted to perform nested PCR amplification of EGFR 21 exon. The primers and nested-PCR protocol were shown in supplement table $1 \mathrm{~S}^{20}$. Briefly, for external PCR amplification, the 25ul reaction contained 10ul single cell lysate, 0.5ul primers(Takara) and 1x GoTaq Colorless Master Mix(Promega). For internal PCR amplification, the 25ul reaction consisted of 0.1 ul product of external PCR amplification, 10ul RNA-free water, 0.5 internal primers and 1x GoTaq Colorless Master Mix. Then the single-cell nested PCR products were visualized by $2 \%$ agarose gel electrophoresis. For H1975 cell line, two blank controls were taken for every six H1975 cells. Beside two blank controls, DNA extracted from cancerous tissue for nested PCR amplification was used as positive control, and two single normal cells captured from each section served as negative controls as well.

Table 1S The nested-PCR amplification primers and protocols for EGFR 21 exon

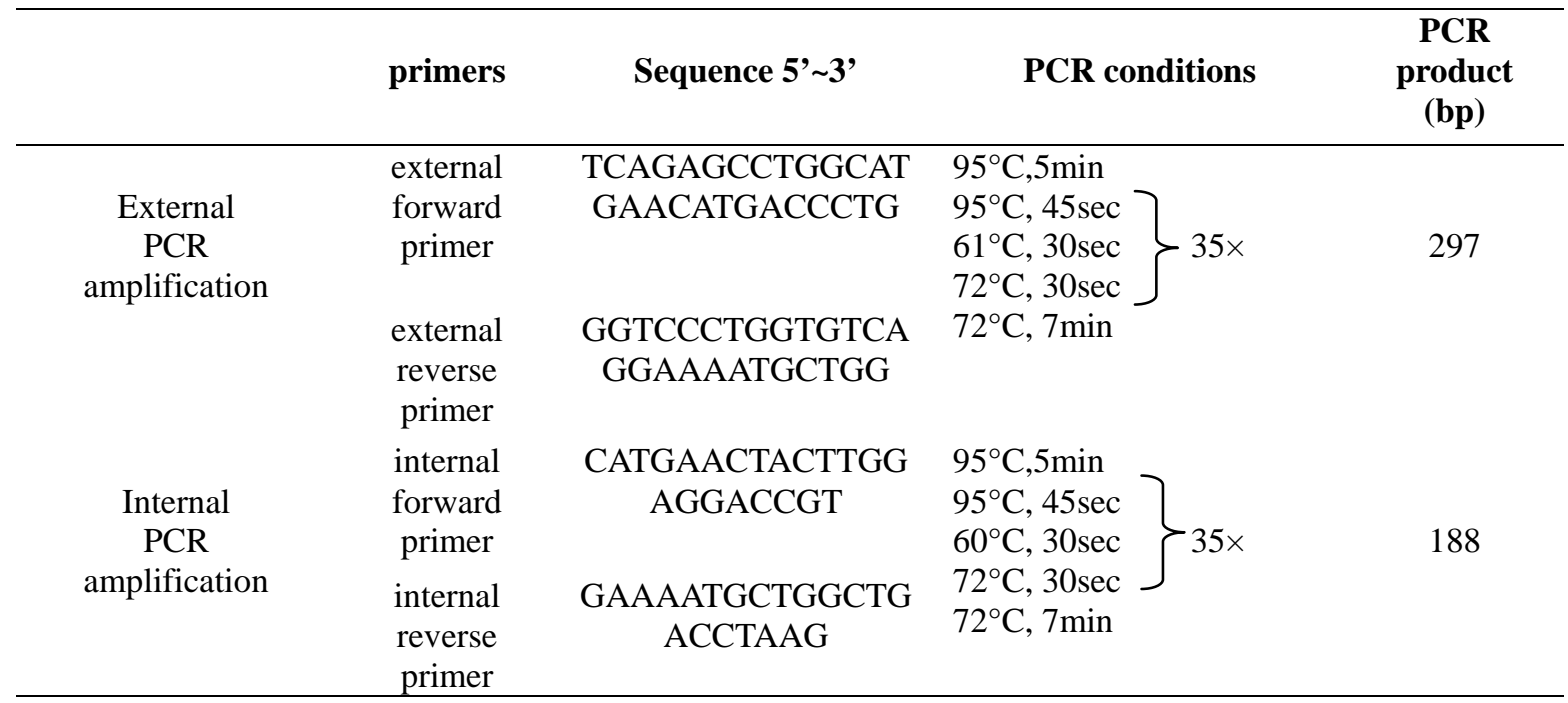




\subsection{EGFR mutation analysis by direct sequencing}

All nested PCR amplified products based on single cell which showed positive PCR reactions by agarose gel electrophoresis were also sequenced to determine EGFR 21 exon status. The products were purified and labeled by using BigDye Terminator v3.1 Cycle Sequencing Kit (Applied Biosystems, Foster City, CA) and then sequenced byABI 3100 Genetic Analyzer (Applied Biosystems). Sequence reactions were read and confirmed by two independent experienced readers.

\subsection{Statistical analysis}

For H1975 cell line, we calculated the efficiency of nested PCR amplification and the rate of allele drop-out(ADO) through direct sequencing.

For tumor samples, the efficiency of nested PCR amplification and the mutational rate in each specimen were computed firstly. Secondly, the nested PCR efficiency and the EGFR mutational rate between two groups were also analyzed and compared by $X^{2}$ test. $\alpha=0.05$ (two-sided) as the difference level, and $P<0.05$ was considered indicative of statistical significance. Statistical analysis was carried out using SPSS 13.0 software (SPSS Inc, USA).

\section{Results}

\subsection{Validation of heterozygous mutation of EGFR in the single cell}

A total of 104 individual H1975 cells were isolated. For individual H1975 cells, the efficiency of nested PCR applied to amplify EGFR 21 exon was 96.2\% (100/104). Supplement figure 1SA showed a picture of agarose gel electrophoresis from nested PCR amplification of single H1975 cells. A total of 93 H1975 cells showed heterozygous mutation of EGFR 21 exon. Four cells were homozygous mutation and the remaining $3 \mathrm{H} 1975$ cells were interpreted as EGFR wild type, indicating allele drop-out (ADO) occurred during the nested PCR process.

In total, 135 tumor cells and 12 normal cells as negative controls were captured by LCM from the six tumor samples in total (Table 2).

Over all six tumor samples, the total success rate was $88.9 \%(120 / 135)$ by nested PCR. The efficiencies in each tumor sample were listed in Table 2. The efficiency of single-cell nested PCR amplification in Group A was 84.3\% (59/70). In Group B the efficiency was 93.8\% (61/65). There was not any significant difference about the efficiency of nested PCR between the two groups $\left(X^{2}=3.119, P=0.077\right)$ (Table 2).

There were no sharp bands detected in all bank controls. For 12 negative controls from single normal cells, 10 normal cells were amplified successfully by nested PCR for EGFR 21 exon. A sharp band were detected in all positive controls. An agarose gel electrophoresis picture of single tumor cells from a tissue section was exhibited in supplement figure 1SB.

Table 2. The efficiencies of nested PCR

\begin{tabular}{cccc}
\hline Patient no. & $\begin{array}{c}\text { Number of } \\
\text { tumor cells }\end{array}$ & $\begin{array}{c}\text { Nested PCR efficiency } \\
\text { (n, \%) }\end{array}$ & P \\
\cline { 1 - 3 } Group A & & $19(82.6 \%)$ & \\
\hline 3647 & 23 & $21(87.5 \%)$ & \\
2715 & 24 & $19(82.6 \%)$ & \\
4128 & 23 & $59(84.3 \% \pm 2.8 \%)$ & \\
total & 70 & $18(90.0 \%)$ & \\
Group B & & $17(91.3 \%)$ & 0.077 \\
3669 & 20 & $22(100.0 \%)$ & \\
1813 & 23 & $61(93.8 \% \pm 5.4 \%)$ & \\
3651 & 22 &
\end{tabular}




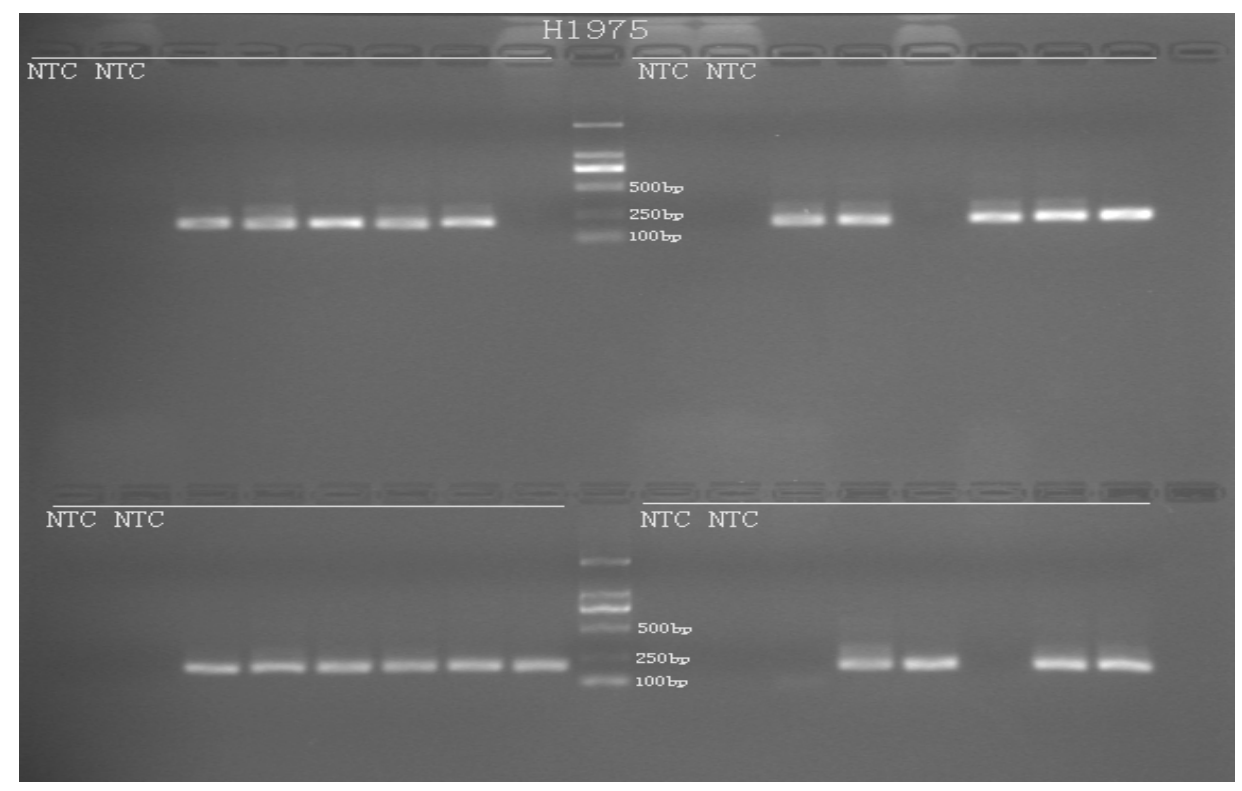

A. Agarose gel electrophoresis from single H1975 cells

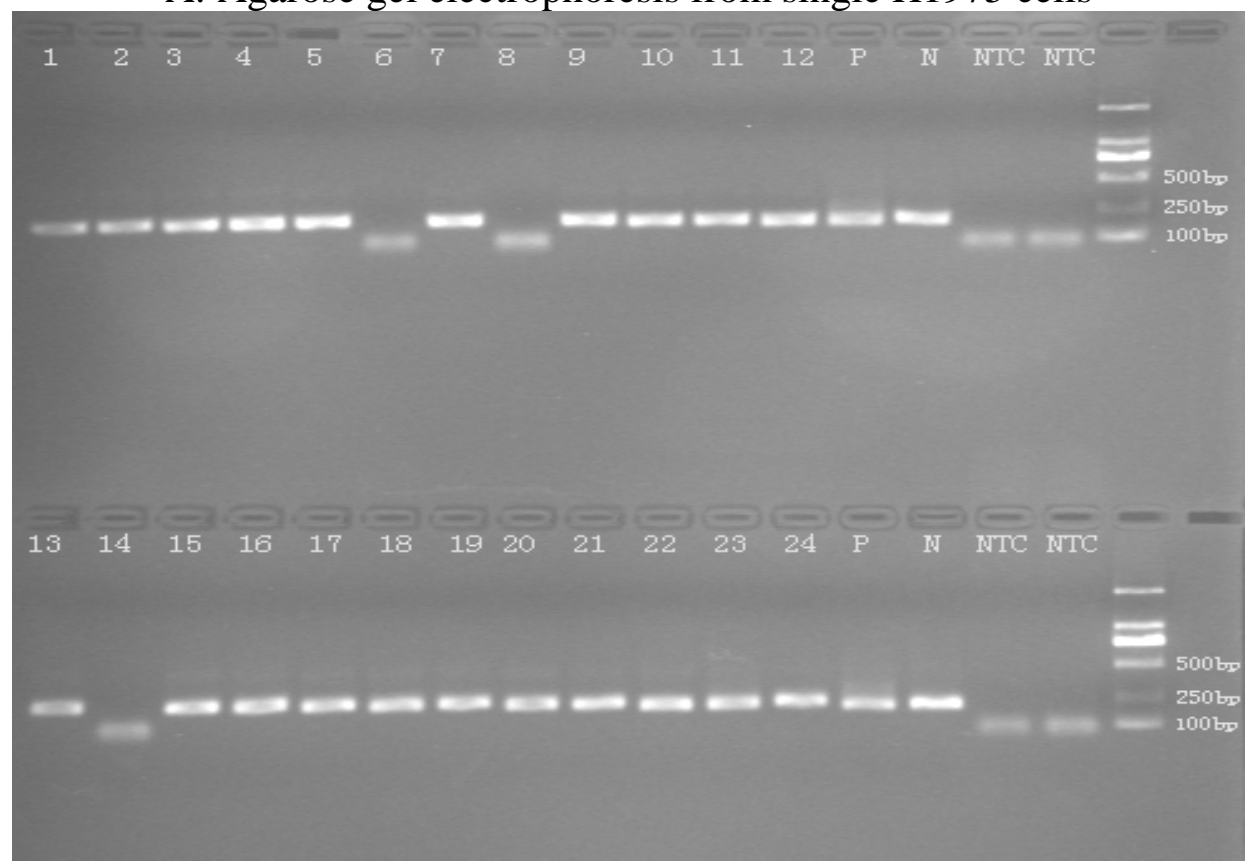

B. Agarose gel electrophoresis from single tumor cells

Figure 1S.The example of agarose gel electrophoresis pictures from single-cell nested PCR amplification.

\subsection{Results of EGFR detection by direct sequencing}

One hundred H1975 cells amplified positive detected by agarose gel electrophoresis were then sequenced to confirm. The success rate was $100 \%(100 / 100)$. Among them, 93 showed EGFR 21 exon L858R heterozygous mutation (Figure 2S A, B). Homogeneous mutation and wild type were detected in 4 cells and 3 cells, respectively. So the rate of allele drop-out (ADO) was 7.0\% (7/100) (Figure 2S C, D). 


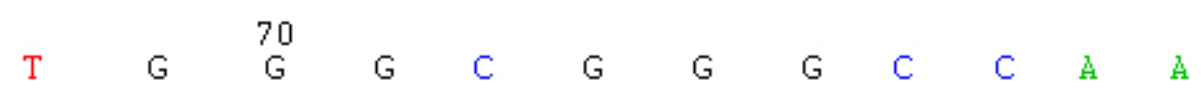
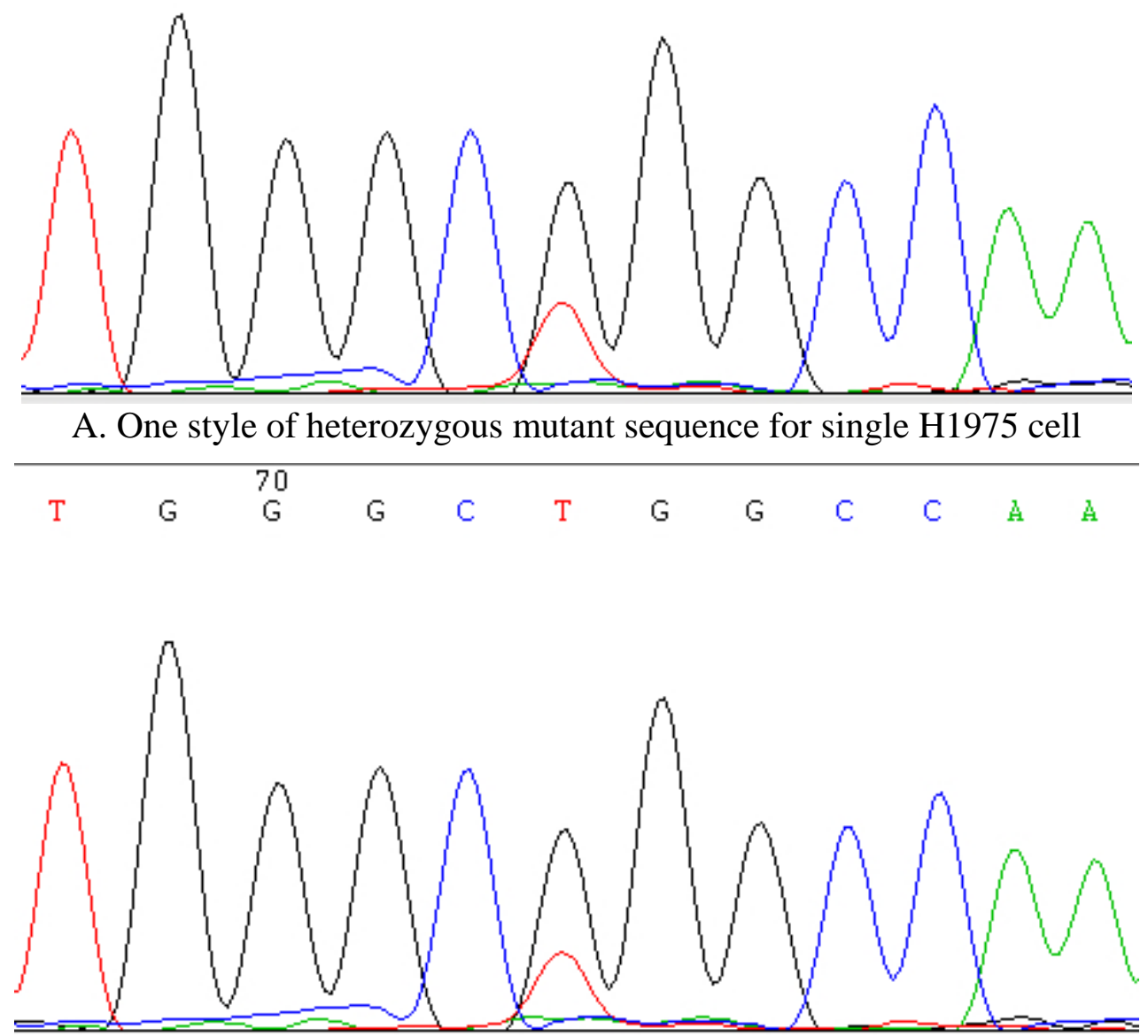

B. The other style of heterozygous mutant sequence for single H1975 cell

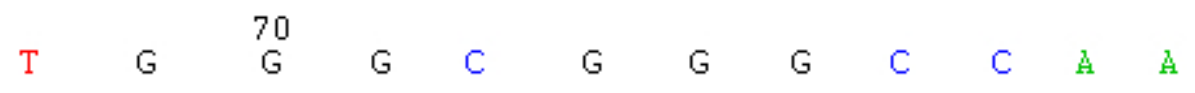

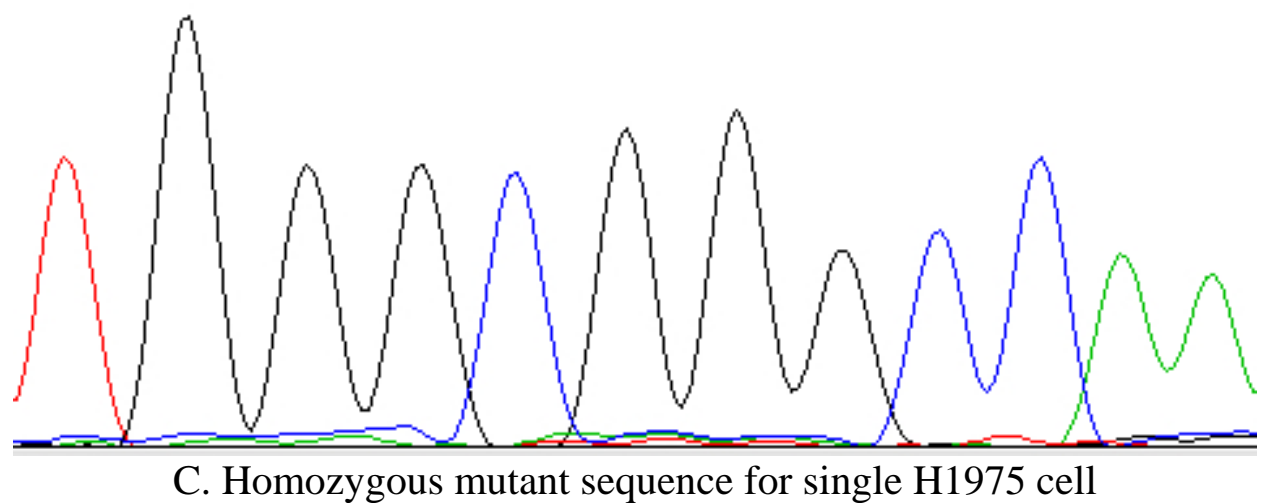

55 


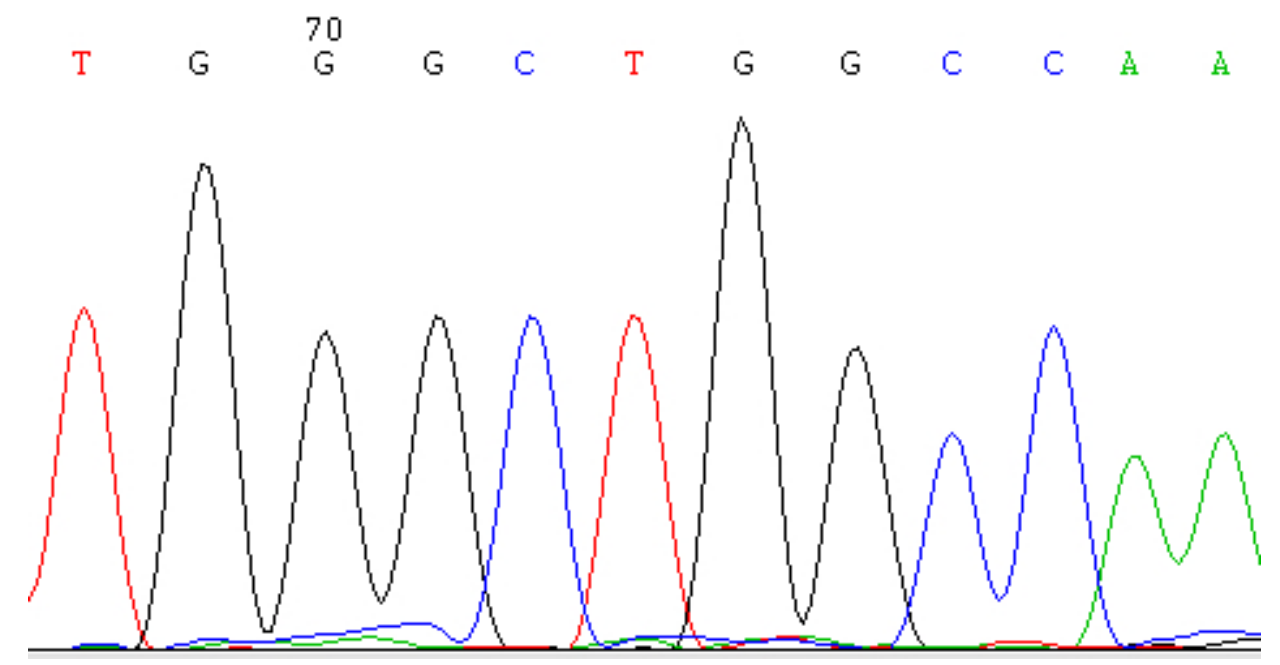

D. Homozygous wild type sequence for single H1975 cell

Figure 2S. Examples of sequencing picture for EGFR 21 exon from single H1975 cells

Among 120 tumor cells amplified successfully, the rate of EGFR 21 exon mutation and wild-type was $77.5 \%(93 / 120)$ and 22.5\%(27/120). The rate of EGFR 21 exon mutation were $86.4 \% \pm 4.9 \%$ and $68.9 \% \pm 2.8 \%$ in Group A and Group B, respectively (Table 3). EGFR 21 exon mutational rate in Group A was higher than that in $\operatorname{Group} \mathrm{B}\left(X^{2}=5.321, P=0.021\right)$.

Table 3.The rate of EGFR 21 exon mutation in each specimen

\begin{tabular}{cccc}
\hline Patient no. & Cells number (n) & mutational rate (n, \%) & P \\
\cline { 1 - 3 } Group A & & & \\
\cline { 1 - 3 } 3647 & 19 & $17(89.5 \%))$ & \\
2715 & 21 & $17(81.0 \%))$ & \\
4128 & 19 & $17(89.5 \%))$ & \\
total & 59 & & \\
Group B & & $13(72.2 \%)$ & \\
3669 & 18 & $14(66.7 \%)$ & \\
1813 & 21 & $15(68.4 \%)$ & \\
3651 & 22 & $42(68.9 \% \pm 2.8 \%)$ & \\
Total & 61 &
\end{tabular}

\section{Discussion}

In the study, H1975 cell line results demonstrated the feasibility of EGFR detection based on single cells. Furthermore, the single-cell analysis demonstrated intratumoral heterogeneity of EGFR activating mutation in lung adenocarcinoma really existed and it might affect the benefit of EGFR-TKI treatment.

In our study, single-cell method described previously was applied to evaluate the heterogeneity of EGFR 21 exon L858R mutation in six adenocarcinoma histologically samples. To our knowledge, no similar studies were designed to evaluate intratumoral heterogeneity of EGFR activating mutations till now. According to our results, EGFR wild-type tumor cells was detected in all the six samples, which suggested the existence of intratumoral heterogeneity for EGFR mutation in lung adenocarcinoma. And EGFR mutational rate in Group A (PFS $>12$ months) was higher than that in Group B (PFS $<6$ months), which indicated that intratumoral heterogeneity of EGFR activating mutations might associated with the response to EGFR-TKIs in the treatment of NSCLC patients with EGFR activating mutation tumors.

Our results were consistent with our previous study and several other studies ${ }^{7,10-12}$, but contrary to Yatabe et al. who reported that intratumoral heterogeneity of EGFR mutation was rare in lung adenocarcinoma $^{13}$. The authors regarded intratumoral EGFR mutational heterogeneity detected as 
psedoheterogeneity resulted from mutant allele specific imbalance(MASI) termed by Gazdar' $\mathrm{s}$ group $^{18,21}$. In some areas within a tumor EGFR is mutated but not amplified. If there was not any normal cells mixed in these areas, the mutational signal would be equivalent to wild-type signal. But a tumor is always mixed with normal cells which might result in EGFR mutated signal diluted and below the threshold of detection. So this situation will lead to pseudoheterogeneous distribution of EGFR mutation in lung cancer. However, without mixture of normal cells the psedoheterogeneity did not happen through the single-cell method in our study. Recently Gerlinger et al also demonstrated the presence of marked intratumoral heterogeneity in regard to somatic mutations in driver and passenger genes ${ }^{22}$. In a word, with the limited number of cases studied, we confirmed EGFR activating mutation was indeed heterogeneous distribution in lung adenocarcinoma.

In the single-cell analysis, ADO is a stochastic and unique problem to PCR of cellular DNA from one cell and can affect either of the alleles of a given locus or strikes at random. It means only one of the two alleles present in a cell is amplified and detected after PCR and then a heterozygous cell will appear homozygosity ${ }^{23}$. Our results suggested ADO led to 7\% error rate in EGFR detection for 21 exon within the range from 5\% to $15 \%$ according to some reports in the field of preimplantation genetic diagnosis (PGD). We also paid attention to contamination of the single-cell method. Blank controls were taken for every 6 single cells tested, and it was encouraging that no non-specifically amplified bands appeared. This demonstrated our procedure for nested PCR amplification did not introduce contaminated DNA. All these results revealed that it was feasible to detect EGFR mutation based on single cells, which laid the foundation for EGFR detection of scare tumor cells from small specimens.

Present study demonstrated that EGFR 21 exon mutational rate in long-term PFS groups was higher than that in short-term PFS groups, which indicating that intratumoral heterogeneity of EGFR activating mutation might affect the benefit of EGFR-TKI treatment. During EGFR-TKI treatment, EGFR mutant tumor cells were inhibited whereas EGFR wild-type tumor cells were trend to proliferation well. Due to this, high EGFR mutational heterogeneity in tumors was easy to resistance to EGFR-TKIs. The result was consistent with our previous study that the relative EGFR mutation abundance in tumor tissues was associated with benefit from EGFR-TKI treatments. However, because of a few samples in present study, we could not make decision the relationship between intratumoral heterogeneity of EGFR activating mutation and benefit of EGFR-TKI treatment in lung adenocarcenoma.

In our study, we selected and detected L858R mutation. Our results indicated that the heterogeneity of L858R have some relationship to the treatment outcome, and to observe the heterogeneity of EGFR, we made a compression between the patients who had longer and shorter PFS. But actually, there was little difference at L858R heterogeneity $(86.4 \% \pm 4.9 \%$ vs. $68.9 \% \pm 2.8 \%$ ). The reason of such situation may because we did not detect the mutations situation of some genes such as T790M and c-met, which could also affect the PFS. In the future study, we will conduct more comprehensive detection of gene mutation, and to investigate the relationship between mutant heteroplasmy and PFS.

There were still some strength and limitations in our study. In order to reduce the reporting and detection bias, we collected and analyzed the PFS data of patients first, and then detected the heterogeneity of EGFR. Also, there were several limitations. Firstly, only one exon of EGFR was detected owing to minute DNA in a single cell. So our study on intratumoral EGFR mutational heterogeneity was only focused on 21 exon of EGFR activating mutation which also inclues 19 exon deletion. According to some reports with the application of whole genome amplification (WGA) sufficient DNA can be obtained to determine EGFR status at multiple sites ${ }^{20,24}$. Secondly, the issue of ADO was inevitable in the process of single-cell PCR amplification which might lead to false-negative result. Although the frequency of ADO evaluated in our study was low, it should be paid an attention. Fluorescent PCR or digital PCR can be used to reduce the occurrence of ADO ${ }^{25}$. Thirdly, the sample size was small. However, the study aimed to the existence of intratumoral EGFR mutational heterogeneity, the relationship between heterogeneity and the therapeutic effect of KTI need large sample validation in the futher suudy. Finally, it was limited for the cases chosen to 
study the controversial problem of intratumoral EGFR mutational heterogeneity. Despite these disadvantadges, our study had provided evidence for the existence of intratumoral heterogeneity for EGFR activating mutation in lung cancer at the level of single cells.

\section{Conclusion}

In conclusion, we demonstrated it is feasible to perform EGFR detection based on single-cell analysis. Then with the application of the method we confirmed the presence of intratumoral EGFR activating mutation heterogeneity in lung adenocarcinoma at the single-cell level. Combined with our previous study, present study suggests that intratumoral heterogeneity in the form of the presence, distribution and abundance of EGFR activating mutations could contribute to plasma ctDNA mutation load and response to EGFR-targeted therapy.

\section{References}

[1] de Groot P, Munden RF. Lung cancer epidemiology, risk factors, and prevention. Radiologic clinics of North America 2012;50:863-876.

[2] Mok TS, Wu YL, Thongprasert S, et al. Gefitinib or carboplatin-paclitaxel in pulmonary adenocarcinoma. The New England journal of medicine 2009;361:947-957.

[3] Mitsudomi T, Morita S, Yatabe Y, et al. Gefitinib versus cisplatin plus docetaxel in patients with non-small-cell lung cancer harbouring mutations of the epidermal growth factor receptor (WJTOG3405): an open label, randomised phase 3 trial. The Lancet Oncology 2010;11:121-128.

[4] Rosell R, Carcereny E, Gervais R, et al. Erlotinib versus standard chemotherapy as first-line treatment for European patients with advanced EGFR mutation-positive non-small-cell lung cancer (EURTAC): a multicentre, open-label, randomised phase 3 trial. The Lancet Oncology 2012;13:239-246.

[5] Zhou C, Wu YL, Chen G, et al. Erlotinib versus chemotherapy as first-line treatment for patients with advanced EGFR mutation-positive non-small-cell lung cancer (OPTIMAL, CTONG-0802): a multicentre, open-label, randomised, phase 3 study. The Lancet Oncology 2011;12:735-742.

[6] Sequist LV, Yang JC, Yamamoto N, et al. Phase III study of afatinib or cisplatin plus pemetrexed in patients with metastatic lung adenocarcinoma with EGFR mutations. Journal of clinical oncology : official journal of the American Society of Clinical Oncology 2013;31:3327-3334.

[7] Zhou Q, Zhang XC, Chen ZH, et al. Relative abundance of EGFR mutations predicts benefit from gefitinib treatment for advanced non-small-cell lung cancer. Journal of clinical oncology : official journal of the American Society of Clinical Oncology 2011;29:3316-3321.

[8] Magee JA, Piskounova E, Morrison SJ. Cancer stem cells: impact, heterogeneity, and uncertainty. Cancer cell 2012;21:283-296.

[9] Jakobsen JN, Sorensen JB. Intratumor heterogeneity and chemotherapy-induced changes in EGFR status in non-small cell lung cancer. Cancer chemotherapy and pharmacology 2012;69:289-299.

[10] Taniguchi K, Okami J, Kodama K, et al. Intratumor heterogeneity of epidermal growth factor receptor mutations in lung cancer and its correlation to the response to gefitinib. Cancer science 2008;99:929-935.

[11] Bai H, Wang Z, Wang Y, et al. Detection and clinical significance of intratumoral EGFR mutational heterogeneity in Chinese patients with advanced non-small cell lung cancer. PloS one 2013;8:e54170.

[12] Chen ZY, Zhong WZ, Zhang XC, et al. EGFR mutation heterogeneity and the mixed response to EGFR tyrosine kinase inhibitors of lung adenocarcinomas. The oncologist 2012;17:978-985. 
[13] Yatabe Y, Matsuo K, Mitsudomi T. Heterogeneous distribution of EGFR mutations is extremely rare in lung adenocarcinoma. Journal of clinical oncology : official journal of the American Society of Clinical Oncology 2011;29:2972-2977.

[14] Mattsson JS, Imgenberg-Kreuz J, Edlund K, et al. Consistent mutation status within histologically heterogeneous lung cancer lesions. Histopathology 2012;61:744-748.

[15] Sun PL, Seol H, Lee HJ, et al. High incidence of EGFR mutations in Korean men smokers with no intratumoral heterogeneity of lung adenocarcinomas: correlation with histologic subtypes, EGFR/TTF-1 expressions, and clinical features. Journal of thoracic oncology : official publication of the International Association for the Study of Lung Cancer 2012;7:323-330.

[16] Ishii S, Tago K, Senoo K. Single-cell analysis and isolation for microbiology and biotechnology: methods and applications. Applied microbiology and biotechnology 2010;86:1281-1292.

[17] Dalerba P, Kalisky T, Sahoo D, et al. Single-cell dissection of transcriptional heterogeneity in human colon tumors. Nature biotechnology 2011;29:1120-1127.

[18] Gandhi J, Zhang J, Xie Y, et al. Alterations in genes of the EGFR signaling pathway and their relationship to EGFR tyrosine kinase inhibitor sensitivity in lung cancer cell lines. PloS one 2009;4:e4576.

[19] Pietersen CY, Lim MP, Woo TU. Obtaining high quality RNA from single cell populations in human postmortem brain tissue. Journal of visualized experiments : JoVE 2009.

[20] Ran R, Li L, Wang M, et al. Determination of EGFR mutations in single cells microdissected from enriched lung tumor cells in peripheral blood. Analytical and bioanalytical chemistry 2013;405:7377-7382.

[21] Soh J, Okumura N, Lockwood WW, et al. Oncogene mutations, copy number gains and mutant allele specific imbalance (MASI) frequently occur together in tumor cells. PloS one 2009;4:e7464.

[22] Gerlinger M, Rowan AJ, Horswell S, et al. Intratumor heterogeneity and branched evolution revealed by multiregion sequencing. The New England journal of medicine 2012;366:883-892.

[23] Piyamongkol W, Bermudez MG, Harper JC, et al. Detailed investigation of factors influencing amplification efficiency and allele drop-out in single cell PCR: implications for preimplantation genetic diagnosis. Molecular human reproduction 2003;9:411-420.

[24] Nelson JR. Random-primed, Phi29 DNA polymerase-based whole genome amplification. Current protocols in molecular biology / edited by Frederick M Ausubel ～[et al] 2014;105:Unit 15 13.

[25] Lee HS, Kim MJ, Ko DS, et al. Preimplantation genetic diagnosis for Charcot-Marie-Tooth disease. Clinical and experimental reproductive medicine 2013;40:163-168. 